Kentaro Takaki

Nagoya Math. J.

Vol. 53 (1974), 71-82

\title{
LIPEOMORPHISMS CLOSE TO AN ANOSOV DIFFEOMORPHISM
}

\author{
KENTARO TAKAKI
}

\section{§ 0. Introduction}

It is well-known that an Anosov diffeomorphism $f$ on a compact manifold is structurally stable in the space of all $C^{1}$-diffeomorphisms, with the $C^{1}$-topology (Anosov [1]). In this paper we show that $f$ is also structurally stable in the space of all lipeomorphisms, with a lipschitz topology. The proof is similar to that of the $C^{1}$-case by J. Moser [4]. If a $C^{1}$-diffeomorphism $g$ is sufficiently close to $f$ in the $C^{1}$-sense $g$ is also sufficiently close to $f$ in the lipschitz sense by the mean value theorem. Hence our result is somewhat stronger than that of Anosov.

In the following let $M$ be a compact connected boundaryless $C^{\infty}$ manifold of dimension $n$ with a Riemannian metric $\|\cdot\|, d$ the distance function induced by $\|\cdot\|$, and $\left\{\left(U_{\alpha}, \alpha\right)\right\}$ a covering of $M$ by finite charts $M=\cup_{\alpha} U_{\alpha}$, where each local diffeomorphism $\alpha$ onto an open subset of $\boldsymbol{R}^{n}$ is defined on an open subset of $M$ which contains the closure of $U_{\alpha}$ : $\mathscr{D}(\alpha) \supset \bar{U}_{\alpha}(\mathscr{D}(\alpha)$ denotes the domain of $\alpha$.). Let $|\cdot|$ be the standard norm in $\boldsymbol{R}^{n}$.

\section{§1. Lipschitz maps on $M$.}

Let $C^{0}(M)$ be the set of all continuous maps of $M$ into itself and $d_{0}$ the distance function on $C^{0}(M)$ induced by the distance function $d$ on $M: d_{0}(f, g)=\operatorname{Sup}_{x \in M} d(f(x), g(x))$ for $f, g \in C^{0}(M)$. $L(M)$ denotes the set of all lipschitz maps of $M$ into itself. It is clear that $L(M)$ is contained in $C^{0}(M)$. We may choose a positive number $\lambda_{1}$ such that for any $\alpha$ $f\left(\bar{U}_{\alpha}\right) \subset \mathscr{D}(\alpha)$ holds for $f \in C^{0}(M)$ with $d_{0}\left(f, 1_{M}\right)<\lambda_{1}, 1_{M}$ denoting the identity map of $M$. For any $f \in C^{0}(M)$ with $d_{0}\left(f, 1_{M}\right)<\lambda_{1}, f$ is lipschitz if and only if for any $\alpha$ the map $\alpha \circ f \circ \alpha^{-1}$ of $\alpha\left(U_{\alpha}\right)$ into $\boldsymbol{R}^{n}$ is lipschitz i.e. the lipschitz constant of $\alpha \circ f \circ \alpha^{-1}: \alpha\left(U_{\alpha}\right) \rightarrow \boldsymbol{R}^{n}$, which is denoted by

Received June 29, 1973. 
$L\left(\alpha \circ f \circ \alpha^{-1}\right.$ on $\left.\alpha\left(U_{\alpha}\right)\right)$ or simply by $L\left(\alpha \circ f \circ \alpha^{-1}\right)$, is finite. This follows from the facts that we can choose a positive number $\rho_{1}$ such that for each $x$ the closed $\rho_{1}$-ball $B\left(x: \rho_{1}\right)=\left\{y \in M \mid d(x, y) \leqq \rho_{1}\right\}$ around $x$ is contained in some $U_{\alpha}$ and that for any chart $(V, \gamma)$ for $M$, and for each compact subset $X$ of $M$ contained in $V$ the map $\gamma:(X, d) \rightarrow(\gamma(X),|\cdot|)$ is a lipeomorphism. We have the following

Proposition 1-1. There exists a positive number $C_{1}$ with the following property: For each $\alpha$ and each $x, y \in U_{\alpha}$ we have $C_{1}^{-1}|\alpha(x)-\alpha(y)| \leqq$ $d(x, y) \leqq C_{1}|\alpha(x)-\alpha(y)|$.

For each $f \in L(M)$ with $d_{0}\left(f, 1_{M}\right)<\lambda_{1}$ we define $d_{\ell}\left(f, 1_{M}\right)$ by $d_{\ell}\left(f, 1_{M}\right)$ $=d_{0}\left(f, 1_{M}\right)+\operatorname{Sup}_{\alpha} L\left(\alpha \circ f \circ \alpha^{-1}-1\right.$ on $\left.\alpha\left(U_{\alpha}\right)\right)$.

Proposition 1-2. Let $f$ be any element in $L(M)$ with $d_{0}\left(f, 1_{M}\right)<\lambda_{1}$. If $d_{\ell}\left(f, 1_{M}\right)$ is sufficiently small $f$ is a lipeomorphism.

Proof. We use the following

LEMMA (Lipschitz Inverse Function Theorem [3]). Let $E, F$ be Banach space, $U \subset E$ and $V \subset F$ non-empty open sets and $g: U \rightarrow V$ a homeomorphism such that $g^{-1}$ is lipschitz. Then for each $h: U \rightarrow F$ with $L(h-g) \cdot L\left(g^{-1}\right)<1, h(U)=V^{\prime}$ is an open set of $F, h: U \rightarrow V^{\prime}$ is a homeomorphism and $h^{-1}: V^{\prime} \rightarrow U$ is lipschitz.

Let $f$ be an element of $L(M)$ such that $d_{0}\left(f, 1_{M}\right)<\lambda_{1}$ and $d_{\ell}\left(f, 1_{M}\right)$ $<\operatorname{Min}\left\{1, \rho_{1} / 2\right\}$. By the above lemma and Prop 1-1 $f\left(U_{\alpha}\right)$ is an open set of $M$ and $f: U_{\alpha} \rightarrow f\left(U_{\alpha}\right)$ is a lipeomorphism. In particular $f(M)$ is open. Since $M$ is compact connected $f(M)=M$. We can complete the proof by proving that $f$ is injective. To do this, take $x, y \in M$ with $f(x)=f(y)$. Then, $d(f(x), x) \leqq d_{0}\left(f, 1_{M}\right) \leqq d_{\ell}\left(f, 1_{M}\right)<\rho_{1} / 2$. Similarly $d(f(y), y)<\rho_{1} / 2$. Hence $y$ is contained in $B\left(x: \rho_{1}\right)$ which is contained in some $U_{\alpha}$. As $f: U_{\alpha} \rightarrow f\left(U_{\alpha}\right)$ is injective we have $x=y$. q.e.d.

\section{§ 2. Lipschitz vector fields on $M$.}

Let $X^{0}(M)$ denote the set of all continuous vector fields on $M$ and $\|\cdot\|$ be the norm on $X^{0}(M)$ induced by the Riemannian metric $\|\cdot\|:\|u\|=$ $\operatorname{Sup}_{x \in M}\left\|u_{x}\right\|$ for any $u=\left(u_{x}\right)_{x \in M} \in X^{0}(M) . \quad\left(X^{0}(M),\|\cdot\|\right)$ is a Banach space. For each $\left(U_{\alpha}, \alpha\right)$ put $U_{\alpha}^{\prime}=\alpha\left(U_{\alpha}\right)$ and let $T_{\alpha}: T M \mid U_{\alpha} \rightarrow U_{\alpha}^{\prime} \times R^{n}$ be the isomorphism induced by $\alpha$. Let $D \alpha: T M \mid U_{\alpha} \rightarrow R^{n}$ be the composite of 
$T_{\alpha}: T M \mid U_{\alpha} \rightarrow U_{\alpha}^{\prime} \times \boldsymbol{R}^{n}$ and the projection $U_{\alpha}^{\prime} \times \boldsymbol{R}^{n} \rightarrow \boldsymbol{R}^{n} . \quad D \alpha$ is considered as the differential of $\alpha$. Then for each $v \in X^{0}(M)$ we define $v_{\alpha}$ by $v_{\alpha}=$ $D \alpha \circ v: U_{\alpha} \rightarrow R^{n}$, and define $|v|$ by $|v|=\operatorname{Sup}_{\alpha} \operatorname{Sup}_{x \in U_{\alpha}}\left|v_{\alpha}(x)\right|$. Then $|\cdot|$ : $X^{0}(M) \rightarrow R^{+}=\{a \in R \mid a \geqq 0\}$ is a norm on $X^{0}(M)$ and it is equivalent to $\|\cdot\|$. The equivalence of $|\cdot|$ and $\|\cdot\|$ follows from the following.

Proposition 2-1. There exists a positive number $C_{2}$ such that for any $\alpha$ and any $v \in T M \mid U_{\alpha}$ we have $C_{2}^{-1}\|v\| \leqq|D \alpha(v)| \leqq C_{2}\|v\|$.

An element $v \in X^{0}(M)$ is called a lipschitz vector field on $M$ if and only if for each $\alpha, v_{\alpha}: U_{\alpha} \rightarrow \boldsymbol{R}^{n}$ is lipschitz i.e. $v_{\alpha} \circ \alpha^{-1}: U_{\alpha}^{\prime} \rightarrow \boldsymbol{R}^{n}$ is lipschitz. Denote the set of all lipschitz vector fields by $X_{\ell}(M)$. We define a norm $|\cdot|_{\ell}$ on $X_{\ell}(M)$ by $|v|_{\ell}=|v|+\operatorname{Sup}_{\alpha}\left\{L\left(v_{\alpha} \circ \alpha^{-1}\right)\right\}$ for any $v \in X_{\ell}(M)$. Then $\left(X_{\ell}(M),|\cdot|_{\ell}\right)$ is a Banach space.

Let $\exp =\left(\exp _{x}\right)_{x \in M}$ be the exponential map induced by the Riemannian metric $\|\cdot\|$. In a normed space $(E,\|\cdot\|)$ we denote the closed $\lambda$-ball around the origin by $(E,\|\cdot\|)_{\lambda}$ and the open $\lambda$-ball around the origin by $(E,\|\cdot\|)_{\lambda}^{\circ}$. We can choose a positive number $\lambda_{2}$ such that for each $x \in M \exp _{x}$ is a diffeomorphism of $\left(T_{x}(M),\|\cdot\|\right)_{\lambda_{2}}^{\circ}$ onto the open $\lambda_{2}$-ball around $x$ in $(M, d)$. Hence for this $\lambda_{2} \exp :\left(X^{0}(M),\|\cdot\|\right)_{\lambda_{2}}^{\circ} v \rightarrow \exp v=\exp \circ v \in\left\{f \in C^{0}(M) \mid\right.$ $\left.d_{0}\left(f, 1_{M}\right)<\lambda_{2}\right\}$ is a bijective map. And for each $v \in\left(X^{0}(M),\|\cdot\|\right)_{\lambda_{1}}^{\circ}$ we have $d_{0}\left(\exp v, 1_{M}\right)=\|v\|$. For the convenience assume $\lambda_{2} \leqq \lambda_{1}$. By the equivalence of $|\cdot|$ and $\|\cdot\|$ we can choose a positive number $\varepsilon_{1}$ such that $\left(X^{0}(M),|\cdot|\right)_{\varepsilon_{1}}^{\circ}$ is contained in $\left(X^{0}(M),\|\cdot\|\right)_{\lambda_{2}}^{\circ}$.

Proposition 2-2. We can choose a positive number $\varepsilon_{2}: 0<\varepsilon_{2} \leqq \varepsilon_{1}$ such that

(i) for each $v \in(M,|\cdot|)_{s_{2}}^{\circ} \exp v$ is contained in $L(M)$ if and only if $v$ is contained in $X_{\ell}(M)$ and that

(ii) for each sequence $\left\{v^{(i)}\right\}_{i=1}^{\infty} \subset X_{\ell}(M) \cap\left(X^{0}(M),|\cdot|\right)_{\varepsilon_{2}}^{\circ}$

$$
d_{\ell}\left(\exp v^{(i)}, 1_{M}\right) \rightarrow 0 \quad \text { as } \quad i \rightarrow \infty,
$$

iff

$$
\left|v^{(i)}\right|_{\ell} \rightarrow 0 \quad \text { as } \quad i \rightarrow \infty
$$

Proof. We take any $\left(U_{\alpha}, \alpha\right)$ and fix it. For each $\left(x^{\prime}, \xi\right) \in U_{\alpha}^{\prime} \times \boldsymbol{R}^{n}$ with $|\xi|<\varepsilon_{1}$ we define $e\left(x^{\prime}, \xi\right)$ by $e\left(x^{\prime}, \xi\right)=\alpha \circ \exp \circ T \alpha^{-1}\left(x^{\prime}, \xi\right)$. By the choice of $\varepsilon_{1}$ this is well-defined and $e$ is of class $C^{\infty}$. Since $e\left(x^{\prime}, 0\right)=x^{\prime}$ 
and $(D e)_{2\left(x^{\prime}, 0\right)}=1_{R^{n}}$, if we represent $e\left(x^{\prime}, \xi\right)$ by $e\left(x^{\prime}, \xi\right)=x^{\prime}+\xi+r\left(x^{\prime} \xi\right)$, then $r$ is of class $C^{\infty}$ and $(D r)_{\left(x^{\prime}, 0\right)}=0$ as $(D r)_{1\left(x^{\prime}, 0\right)}=(D r)_{2\left(x^{\prime}, 0\right)}=0$ for any $x^{\prime} \in U_{\alpha}^{\prime}$. Recalling that $\mathscr{D}(\alpha) \supset \bar{U}_{\alpha}$, by the mean value theorem, we have the following

(A): There exist a positive number $\varepsilon_{2}^{(\alpha)}: 0<\varepsilon_{2}^{(\alpha)} \leqq \varepsilon_{1}$ and a function $L^{(\alpha)}:\left(0, \varepsilon_{2}^{(\alpha)}\right) \rightarrow[0,1)$ satisfying the following properties.

(iii) For each $x^{\prime}, y^{\prime} \in U_{\alpha}^{\prime}, 0<\varepsilon<\varepsilon_{2}^{(\alpha)}$ and $\xi, \eta \in \boldsymbol{R}^{n}$ with $|\xi|,|\eta| \leqq \varepsilon$ we have $\left|r\left(x^{\prime}, \xi\right)-r\left(y^{\prime}, \eta\right)\right| \leqq L^{(\alpha)}(\varepsilon)\left\{\left|x^{\prime}-y^{\prime}\right|+|\xi-\eta|\right\}$.

(iv) $L^{(\alpha)}(\varepsilon) 0$ as $\varepsilon \rightarrow 0$

Now, take $\varepsilon: 0<\varepsilon<\varepsilon_{2}^{(\alpha)}$ and $v \in\left(X^{0}(M),|\cdot|\right)_{s}$ and put $v_{\alpha}=D \alpha \circ v: U_{\alpha} \rightarrow \boldsymbol{R}^{n}$ and $h=\exp v \in C^{0}(M)$. We have $h\left(\bar{U}_{\alpha}\right) \subset \mathscr{D}(\alpha)$ since $d_{0}\left(h, 1_{M}\right)=\|v\|<\lambda_{2} \leqq \lambda_{1}$.

For each $x^{\prime} \in U_{\alpha}^{\prime}$ put $x=\alpha^{-1}\left(x^{\prime}\right)$. Then, we have

$$
\begin{aligned}
\left(x^{\prime}, v_{\alpha} \circ \alpha^{-1}\left(x^{\prime}\right)\right) & =T \alpha\left(v_{x}\right)=T \alpha \circ \exp _{x}^{-1}(h(x)) \\
& =T \alpha \circ \exp _{x}^{-1} \circ \alpha^{-1}\left(\alpha \circ h \circ \alpha^{-1}\left(x^{\prime}\right)\right),
\end{aligned}
$$

which implies

$$
\begin{aligned}
\alpha \circ h \circ \alpha^{-1}\left(x^{\prime}\right) & =e\left(x^{\prime}, v_{\alpha} \circ \alpha^{-1}\left(x^{\prime}\right)\right) \\
& =x^{\prime}+v_{\alpha} \circ \alpha^{-1}\left(x^{\prime}\right)+r\left(x^{\prime}, v_{\alpha} \circ \alpha^{-1}\left(x^{\prime}\right)\right),
\end{aligned}
$$

from which we get

$$
\left(\alpha \circ h \circ \alpha^{-1}-1\right)\left(x^{\prime}\right)=v_{\alpha} \circ \alpha^{-1}\left(x^{\prime}\right)+r\left(x^{\prime}, v_{\alpha} \circ \alpha^{-1}\left(x^{\prime}\right)\right) .
$$

Hence for each $x^{\prime}, y^{\prime} \in U_{\alpha}^{\prime}$ we have

$$
\begin{aligned}
& \left(\alpha \circ h \circ \alpha^{-1}-1\right)\left(x^{\prime}\right)-\left(\alpha \circ h \circ \alpha^{-1}-1\right)\left(y^{\prime}\right) \\
& \quad=\left\{v_{\alpha} \circ \alpha^{-1}\left(x^{\prime}\right)-v_{\alpha} \circ \alpha^{-1}\left(y^{\prime}\right)\right\}+\left\{r\left(x^{\prime}, v_{\alpha} \circ \alpha^{-1}\left(x^{\prime}\right)\right)-r\left(y^{\prime}, v_{\alpha} \circ \alpha^{-1}\left(y^{\prime}\right)\right)\right\} .
\end{aligned}
$$

By this equality we have the followings:

( $v$ ) If $v$ is lipschitz then we have

$$
\begin{aligned}
&\left|\left(\alpha \circ h \circ \alpha^{-1}-1\right)\left(x^{\prime}\right)-\left(\alpha \circ h \circ \alpha^{-1}-1\right)\left(y^{\prime}\right)\right| \\
& \leqq L\left(v_{\alpha} \circ \alpha^{-1}\right)\left|x^{\prime}-y^{\prime}\right|+L^{(\alpha)}(\varepsilon)\left\{\left|x^{\prime}-y^{\prime}\right|+L\left(v_{\alpha} \circ \alpha^{-1}\right)\left|x^{\prime}-y^{\prime}\right|\right\} \\
& \leqq\left\{L^{(\alpha)}(\varepsilon)+|v|_{\ell}+L^{(\alpha)}(\varepsilon)|v|_{\ell}\right\}\left|x^{\prime}-y^{\prime}\right| .
\end{aligned}
$$

(vi) If $h=\exp v$ is lipschitz then we have

$$
\begin{aligned}
d_{\ell}\left(h, 1_{M}\right) \cdot\left|x^{\prime}-y^{\prime}\right| & \geqq L\left(\alpha \circ h \circ \alpha^{-1}-1\right)\left|x^{\prime}-y^{\prime}\right| \\
& \geqq\left|\left(\alpha \circ h \circ \alpha^{-1}-1\right)\left(x^{\prime}\right)-\left(\alpha \circ h \circ \alpha^{-1}-1\right)\left(y^{\prime}\right)\right| \\
& \geqq\left|v_{\alpha} \circ \alpha^{-1}\left(x^{\prime}\right)-v_{\alpha} \circ \alpha^{-1}\left(y^{\prime}\right)\right|
\end{aligned}
$$




$$
\begin{aligned}
& -\left|r\left(x^{\prime}, v_{\alpha} \circ \alpha^{-1}\left(x^{\prime}\right)\right)-r\left(y^{\prime}, v_{\alpha} \circ \alpha^{-1}\left(y^{\prime}\right)\right)\right| \\
\geqq & \left|v_{\alpha} \circ \alpha^{-1}\left(x^{\prime}\right)-v_{\alpha} \circ \alpha^{-1}\left(y^{\prime}\right)\right| \\
& -L^{(\alpha)}(\varepsilon)\left\{\left|x^{\prime}-y^{\prime}\right|+\left|v_{\alpha} \circ \alpha^{-1}\left(x^{\prime}\right)-v_{\alpha} \circ \alpha^{-1}\left(y^{\prime}\right)\right|\right\}
\end{aligned}
$$

As $0 \leqq L^{(\alpha)}(\varepsilon)<1$ we have by this inequality

$$
\begin{aligned}
& \left|v_{\alpha} \circ \alpha^{-1}(x)-v_{\alpha} \circ \alpha^{-1}(y)\right| \\
& \quad \leqq\left[\left\{d_{\ell}\left(h, 1_{M}\right)+L^{(\alpha)}(\varepsilon)\right\} /\left(1-L^{(\alpha)}(\varepsilon)\right)\right] \cdot\left|x^{\prime}-y^{\prime}\right|
\end{aligned}
$$

The proof is complete by using (iv), (v) and (v).

q.e.d.

\section{§3. Lipeomorphisms close to an Anosov diffeomorphism on $M$.}

LEMMA 3-1. There exist positive numbers $\varepsilon_{3}: 0<\varepsilon_{3} \leqq \varepsilon_{1}$ and $C_{3}$ with the following property. For any $x \in U_{\alpha}$ and $\xi, \eta \in \boldsymbol{R}^{n}$ with $|\xi|,|\eta|<\varepsilon_{3}$ we have

$$
C_{3}^{-1}|\xi-\eta| \leqq\left|y^{\prime}-z^{\prime}\right| \leqq C_{3}|\xi-\eta|
$$

where $y^{\prime}=\alpha \circ \exp _{x} \circ(D \alpha)_{x}^{-1}(\xi)$ and $z^{\prime}=\alpha \circ \exp _{x} \circ(D \alpha)_{x}^{-1}(\eta)$.

Proof. Take $\alpha$ and fix it. In the proof of Prop. 2-2 we defined $e$ and $r$. By (A) we can choose a positive number $\varepsilon_{3}^{(\alpha)}: 0<\varepsilon_{3}^{(\alpha)} \leqq \varepsilon_{1}$ such that for any $x^{\prime}, y^{\prime} \in U_{\alpha}^{\prime}$ and any $\xi, \eta \in \boldsymbol{R}^{n}$ with $|\xi|,|\eta|<\varepsilon_{3}^{(\alpha)}$ we have

$$
\left|r\left(x^{\prime}, \xi\right)-r\left(y^{\prime}, \eta\right)\right| \leqq 1 / 2\left(\left|x^{\prime}-y^{\prime}\right|+|\xi-\eta|\right)
$$

For any $x \in U_{\alpha}$ and $\xi, \eta \in \boldsymbol{R}^{n}$ with $|\xi|,|\eta|<\varepsilon_{3}^{(\alpha)}$ putting $y^{\prime}=\alpha \circ \exp _{x} \circ(D \alpha)_{x}^{-1}(\xi)$, $z^{\prime}=\alpha \circ \exp _{x} \circ(D \alpha)_{x}^{-1}(\eta)$ and $x^{\prime}=\alpha(x)$, we have $y^{\prime}=e\left(x^{\prime}, \xi\right)$ and $z^{\prime}=e\left(x^{\prime}, \eta\right)$.

Hence

$$
\begin{aligned}
\left|y^{\prime}-z^{\prime}\right| & \leqq|\xi-\eta|+\left|r\left(x^{\prime}, \xi\right)-r\left(x^{\prime}, \eta\right)\right| \leqq|\xi-\eta|+1 / 2|\xi-\eta| \\
& \leqq C_{3}|\xi-\eta|
\end{aligned}
$$

and

$$
\begin{aligned}
\left|y^{\prime}-z^{\prime}\right| & \geqq|\xi-\eta|-\left|r\left(x^{\prime}, \xi\right)-r\left(x^{\prime}, \eta\right)\right| \geqq|\xi-\eta|-1 / 2|\xi-\eta| \\
& \geqq C_{3}^{-1}|\xi-\eta|
\end{aligned}
$$

Hence we can take $C_{3}=2$ and $\varepsilon_{3}=\operatorname{Inf}_{\alpha}\left\{\varepsilon_{3}^{(\alpha)}\right\}$

q.e.d.

CoRollary. We can take positive numbers $\lambda$ and $C$ such that for any $x \in M$ and $u, v \in T_{x} M$ with $\|u\|,\|v\|<\lambda$ we have

$$
C^{-1}\|u-v\| \leqq d\left(\exp _{x} u, \exp _{x} v\right) \leqq C\|u-v\| .
$$


Proof. This follows from Lemma 3-1, Prop. 1-1 and Prop. 2-1.

q.e.d.

LEMMA 3-2. There exist positive numbers $\delta_{1}, \varepsilon_{4}: 0<\varepsilon_{4} \leqq \varepsilon_{3}$, a function $L_{1}:\left(0, \delta_{1}\right) \times\left(0, \varepsilon_{4}\right) \rightarrow R^{+}$and a continuous map $r:\left(X_{\ell}(M),|\cdot|_{\ell}\right)_{\delta_{1}}^{\circ} \times\left(X^{0}(M),|\cdot|\right)_{\varepsilon_{4}}^{\circ}$ $\rightarrow X^{0}(M)$ with the following properties:

(i) It holds that $\exp w \circ \exp v=\exp (w+v+r(w, v))$ for each $w \in\left(X_{\ell}(M),|\cdot|_{\ell}\right)_{\dot{o}_{1}}^{\circ}$ and $v \in\left(X^{0}(M),|\cdot|\right)_{\varepsilon_{4}}^{\circ}$

(ii) For each $\delta: 0<\delta<\delta_{1}, \varepsilon: 0<\varepsilon<\varepsilon_{4}, w \in\left(X_{\ell}(M),|\cdot|_{\ell}\right)_{\delta}$ and $v, v^{\prime} \in\left(X^{0}(M),|\cdot|\right)_{\varepsilon}$ we have $\left|r(w, v)-r\left(w, v^{\prime}\right)\right| \leqq L_{1}(\delta, \varepsilon)\left|v-v^{\prime}\right|$ and $r(w, 0)=r(0, v)=0$.

(iii) $L_{1}(\delta, \varepsilon) \rightarrow 0$ as $\delta, \varepsilon \rightarrow 0$.

Proof. Choose open subsets $V_{\alpha}$ of $M$ for each $\alpha$ such that $V_{\alpha} \subset \bar{V}_{\alpha}$ $\subset U_{\alpha}$ and $\cup_{\alpha} V_{\alpha}=M$. We define a norm $|\cdot|^{\prime}$ on $X^{0}(M)$ with respect to the covering by finite charts, $\left\{\left(V_{\alpha}, \alpha\right)\right\}_{\alpha}$, in the same way as we defined |. |: For each $v \in X^{0}(M)$ we define $|v|^{\prime}$ by $|v|^{\prime}=\operatorname{Sup}_{\alpha} \operatorname{Sup}_{x \in V_{\alpha}}\left|v_{\alpha}(x)\right|$, where $v_{\alpha}=D \alpha \circ v$. As $\mid \cdot \gamma^{\prime}$ and $\|\cdot\|$ are equivalent $\mid \cdot \gamma^{\prime}$ and $|\cdot|$ are equivalent. We can choose a positive number $\varepsilon_{4}^{\prime}: 0<\varepsilon_{4}^{\prime} \leqq \varepsilon_{3}$ such that for any $w$, $v \in X^{0}(M)$ with $|w|,|v|^{\prime}<\varepsilon_{4}^{\prime}$ we have $\exp v\left(\bar{V}_{\alpha}\right) \subset U_{\alpha}$ for any $\alpha$ and $d_{0}\left(\exp w \circ \exp v, 1_{M}\right)<\lambda_{2}$. Then for each $w, v \in X^{0}(M)$ with $|w|,|v|^{\prime}<\varepsilon_{4}^{\prime}$ there exists a unique $r(w, v) \in X^{0}(M)$ such that $\exp w \circ \exp v=\exp (w+$ $v+r(w, v))$ and $d_{0}\left(\exp w \circ \exp v, 1_{M}\right)=\|w+v+r(w, v)\|$. It is clear that $r$ is continuous and $r(w, 0)=(0, v)=0$. Take any $\alpha$ and fix it. Put $V_{\alpha}^{\prime}=\alpha\left(V_{\alpha}\right)$. For each $\left(x^{\prime}, \xi, \eta\right) \in V_{\alpha}^{\prime} \times \boldsymbol{R}^{n} \times \boldsymbol{R}^{n}$ with $|\xi|,|\eta|<\varepsilon_{4}^{\prime}$ we define $P_{\alpha}\left(x^{\prime}, \xi, \eta\right)$ by $P_{\alpha}\left(x^{\prime}, \xi, \eta\right)=D_{\alpha} \circ \exp _{x}^{-1} \circ \exp _{y} \circ(D \alpha)_{y}^{-1}(\xi)$, where $x=\alpha^{-1}\left(x^{\prime}\right)$ and $y=\exp _{x} \circ(D \alpha)_{x}^{-1}(\eta)$. By the choice of $\varepsilon_{4}^{\prime}$ this is well-defined and $P_{\alpha}$ is of class $C^{\infty}$. It is clear that $P_{\alpha}\left(x^{\prime}, 0,0\right)=0, P_{\alpha}\left(x^{\prime}, \xi, 0\right)=\xi$ and $P_{\alpha}\left(x^{\prime}, 0, \eta\right)$ $=\eta$. Hence if we express $P_{\alpha}\left(x^{\prime}, \xi, \eta\right)$ by $P_{\alpha}\left(x^{\prime}, \xi, \eta\right)=\xi+\eta+r^{(\alpha)}\left(x^{\prime}, \xi, \eta\right)$ then $r^{(\alpha)}$ is of class $C^{\infty},\left(D r^{(\alpha)}\right)_{1\left(x^{\prime}, \xi, 0\right)}=\left(D r^{(\alpha)}\right)_{1\left(x^{\prime}, 0, \eta\right)}=0,\left(D r^{(\alpha)}\right)_{2\left(x^{\prime}, \xi, 0\right)}=0$, $\left(D r^{(\alpha)}\right)_{3\left(x^{\prime}, 0, \eta\right)}=0$ and so in particular $\left(D r^{(\alpha)}\right)_{\left(x^{\prime}, 0,0\right)}=0$. Noting that $\mathscr{D}(\alpha) \supset \bar{U}_{\alpha} \supset U_{\alpha} \supset \bar{V}_{\alpha} \supset V_{\alpha}$, we can conclude the following by the mean value theorem.

(B) There exist two positive numbers $\delta_{1}^{\prime}: 0<\delta_{1}^{\prime} \leqq \varepsilon_{4}^{\prime}$ and $\varepsilon_{4}^{\prime \prime}: 0<\varepsilon_{4}^{\prime \prime} \leqq \varepsilon_{4}^{\prime}$ and a function $L_{1}^{(\alpha)}:\left(0, \delta_{1}^{\prime}\right) \times\left(0, \varepsilon_{4}^{\prime \prime}\right) \rightarrow \boldsymbol{R}^{+}$with the following properties:

(iv) For each $\delta: 0<\delta<\delta_{1}^{\prime}, \varepsilon: 0<\varepsilon<\varepsilon_{4}^{\prime \prime}, x^{\prime}, y^{\prime} \in V_{\alpha}^{\prime}$ and $\xi, \eta, \zeta, \theta \in R^{n}$ with $|\xi|,|\zeta| \leqq \delta$ and $|\eta|,|\theta| \leqq \varepsilon$ we have

$$
\left|r^{(\alpha)}\left(x^{\prime}, \xi, \eta\right)-r^{(\alpha)}\left(y^{\prime}, \zeta, \theta\right)\right| \leqq L_{1}^{(\alpha)}(\delta, \varepsilon) \cdot\left\{\left|x^{\prime}-y^{\prime}\right|+|\xi-\zeta|+|\eta-\theta|\right\},
$$

(v) $L_{1}^{(\alpha)}(\delta, \varepsilon) \rightarrow 0$ as $\delta, \varepsilon \rightarrow 0$. 
Take any positive numbers $\delta$, $\varepsilon$ with $0<\delta<\delta_{1}^{\prime}$ and $0<\varepsilon<\varepsilon_{4}^{\prime \prime}$ and fix them. For each $w, v, v^{\prime} \in X^{0}(M)$ with $|w| \leqq \delta$ and $|v|^{\prime},\left|v^{\prime}\right|^{\prime} \leqq \varepsilon$ we define $w_{\alpha}, v_{\alpha}, v_{\alpha}^{\prime}, r(w, v)_{\alpha}$ and $r\left(w, v^{\prime}\right)_{\alpha}$ as before. Then for each $x^{\prime} \in V_{\alpha}^{\prime}$ we have $r(w, v)_{\alpha} \circ \alpha^{-1}\left(x^{\prime}\right)=P_{\alpha}\left(x^{\prime}, w_{\alpha} \circ \alpha^{-1}\left(y^{\prime}\right), v_{\alpha} \circ \alpha^{-1}\left(x^{\prime}\right)\right)-\left\{w_{\alpha} \circ \alpha^{-1}\left(x^{\prime}\right)+v_{\alpha} \circ \alpha^{-1}\left(x^{\prime}\right)\right\}$

and

$$
\begin{aligned}
r\left(w, v^{\prime}\right)_{\alpha} \circ \alpha^{-1}\left(x^{\prime}\right)= & P_{\alpha}\left(x^{\prime}, w_{\alpha} \circ \alpha^{-1}\left(z^{\prime}\right), v_{\alpha}^{\prime} \circ \alpha^{-1}\left(x^{\prime}\right)\right) \\
& -\left\{w_{\alpha} \circ \alpha^{-1}\left(x^{\prime}\right)+v_{\alpha}^{\prime} \circ \alpha^{-1}\left(x^{\prime}\right)\right\},
\end{aligned}
$$

where

$$
x=\alpha^{-1}\left(x^{\prime}\right), \quad y^{\prime}=\alpha \circ \exp _{x} \circ(D \alpha)_{x}^{-1}\left(v_{\alpha} \circ \alpha^{-1}(x)\right)
$$

and

$$
z^{\prime}=\alpha \circ \exp _{x} \circ(D \alpha)_{x}^{-1}\left(v_{\alpha}^{\prime} \circ \alpha^{-1}\left(x^{\prime}\right)\right)
$$

Hence we get

$$
\begin{aligned}
&\left|r(w, v)_{\alpha} \circ \alpha^{-1}\left(x^{\prime}\right)-r\left(w, v^{\prime}\right)_{\alpha} \circ \alpha^{-1}\left(x^{\prime}\right)\right| \\
& \leqq\left|w_{\alpha} \circ \alpha^{-1}\left(y^{\prime}\right)-w_{\alpha} \circ \alpha^{-1}\left(z^{\prime}\right)\right|+\mid r^{(\alpha)}\left(x^{\prime}, w_{\alpha} \circ \alpha^{-1}\left(y^{\prime}\right), v_{\alpha} \circ \alpha^{-1}\left(x^{\prime}\right)\right) \\
& \quad-r^{(\alpha)}\left(x^{\prime}, w_{\alpha} \circ \alpha^{-1}\left(z^{\prime}\right), v_{\alpha}^{\prime} \circ \alpha^{-1}\left(x^{\prime}\right)\right) \mid \\
& \leqq\left\{1+L_{1}^{(\alpha)}(\delta, \varepsilon)\right\} \cdot\left|w_{\alpha} \circ \alpha^{-1}\left(y^{\prime}\right)-w_{\alpha} \circ \alpha^{-1}\left(z^{\prime}\right)\right| \\
&+L_{1}^{(\alpha)}(\delta, \varepsilon)\left|v_{\alpha} \circ \alpha^{-1}\left(x^{\prime}\right)-v_{\alpha}^{\prime} \circ \alpha^{-1}\left(x^{\prime}\right)\right| .
\end{aligned}
$$

If we assume that $w$ is contained in $L(M)$, then we have by Lemma 3-1

$$
\begin{aligned}
&\left|r(w, v)_{\alpha} \circ \alpha^{-1}\left(x^{\prime}\right)-r\left(w, v^{\prime}\right)_{\alpha} \circ \alpha^{-1}\left(x^{\prime}\right)\right| \\
& \leqq\left\{1+L_{1}^{(\alpha)}(\delta, \varepsilon)\right\} \cdot|w|_{\ell} \cdot\left|y^{\prime}-z^{\prime}\right|+L_{1}^{(\alpha)}(\delta, \varepsilon)\left|v_{\alpha} \circ \alpha^{-1}\left(x^{\prime}\right)-v_{\alpha}^{\prime} \circ \alpha^{-1}\left(x^{\prime}\right)\right| \\
& \leqq\left\{L_{1}^{(\alpha)}(\delta, \varepsilon)+C_{3}|w|_{\ell} \cdot\left(1+L_{1}^{(\alpha)}(\delta, \varepsilon)\right)\right\} \cdot\left|v_{\alpha} \circ \alpha^{-1}\left(x^{\prime}\right)-v_{\alpha}^{\prime} \circ \alpha^{-1}\left(x^{\prime}\right)\right|
\end{aligned}
$$

From this inequality, the equivalence of $|\cdot|$ and $\mid \cdot \gamma^{\prime}$ and (v) the proof of Lemma 3-2 is complete.

In the followings we assume that $f: M \rightarrow M$ is a $C^{1}$-diffeomorphism. For this $f$ we define a linear automorphism $f_{*}$ of $X^{0}(M)$ by

$$
f_{*}(v)=d f \circ v \circ f^{-1} \quad \text { for any } \quad v \in X^{0}(M),
$$

where $d f$ is the differential of $f$.

LEMMA 3-3. There exist a positive number $\varepsilon_{5}$, a bounded function $L_{2}:\left(0, \varepsilon_{5}\right) \rightarrow R^{+}$and a continuous map $s:\left(X^{0}(M),|\cdot|\right)_{\varepsilon_{5}}^{\circ} \rightarrow X^{0}(M)$ with the 
following properties.

(i ) $f \circ \exp v \circ f^{-1}=\exp \left(f_{*}(v)+s(v)\right)$ for any $v \in\left(X^{0}(M),|\cdot|\right)_{\varepsilon_{5}}^{\circ}$,

(ii) $s(0)=0$ and for each $\varepsilon: 0<\varepsilon<\varepsilon_{5}$ and $v, v^{\prime} \in\left(X^{0}(M),|\cdot|\right)$, we have

$$
\left|s(v)-s\left(v^{\prime}\right)\right| \leqq L_{2}(\varepsilon)\left|v-v^{\prime}\right|,
$$

(iii) $L_{2}(\varepsilon) \rightarrow 0$ as $\varepsilon \rightarrow 0$.

Proof. (cf. [4]) We can define a map $F$ of a neighborhood of the origin in $X^{0}(M)$ into $X^{0}(M)$ such that $\exp (F(v))=f \circ \exp v \circ f^{-1}$ for each $v \in X^{0}(M)$ with $|v|$ sufficiently small. It is clear that $F(0)=0$. Since $f$ is of class $C^{1}, F$ is so and in fact, the differential of $F$ at the origin is $f_{*}$. Hence the proof is easy by using the mean value theorem for $s=$ $F-f_{*}$.

For the convenience we may assume $\varepsilon_{5} \leqq \varepsilon_{4}$.

Let $X_{b}(M)$ be the set of all bounded vector fields on $M$. A complete norm $\|\cdot\|_{b}$ on $X_{b}(M)$ is defined by

$$
\|v\|_{b}=\operatorname{Sup}_{x \in M}\left\|v_{x}\right\| \text { for any } v \in X_{b}(M) .
$$

Lemma $3-3$ is also true for $\left(X_{b}(M),\|\cdot\|_{b}\right)$. We make use of the same notations as those in Lemma $3-3$ for $\left(X_{b},\|\cdot\|_{b}\right), f_{*}, \varepsilon_{5}, L_{2}, s$. If $f$ is an Anosov deffeomorphism $1-f_{*}$ is a linear automorphism of $X^{0}(M)$ and also of $X_{b}(M)$, where 1 is the identity map (cf. [4]).

We will prove the following well known fact.

LEMMA 3-4. If $f$ is an Anosov diffeomorphism then $f$ is expansive i.e. there exists a positive number $\lambda_{0}$ such that $\operatorname{Sup}_{n \in Z} d\left(f^{n}(x), f^{n}(y)\right)>\lambda_{0}$ for any $x, y \in M$ with $x \neq y$.

Proof. (cf. [5]) By the above remark there exists a positive number $\lambda_{0}: 0<2 \lambda_{0}<\lambda_{2}$ such that for each $v, v^{\prime} \in\left(X_{b}(M),\|\cdot\|_{b}\right)_{2 \lambda_{0}}$ we have

$$
\left\|s(v)-s\left(v^{\prime}\right)\right\|_{b} \leqq 1 / 2 \cdot\left\|\left(1-f_{*}\right)^{-1}\right\|_{b}^{-1} \cdot\left\|v-v^{\prime}\right\|_{b} .
$$

We assert the following.

(C) Let $u$ be a map of $M$ into itself such that $f \circ u=u \circ f$ and $u \neq 1_{M}$. Then $d_{0}\left(u, 1_{M}\right)=\operatorname{Sup}_{x \in M} d(u(x), x)>2 \cdot \lambda_{0}$.

Choose any map $u: M \rightarrow M$ with $f \circ u=u \circ f$ and $d_{0}\left(u, 1_{M}\right) \leqq 2 \cdot \lambda_{0}$. For this $u$ there exists a unique element $v \in X_{b}(M)$ such that $u=\exp v$ and 


$$
d_{0}\left(u, 1_{M}\right)=\|v\|_{b}
$$

Then we have

$$
f \circ \exp v \circ f^{-1}=f \circ u \circ f^{-1}=u=\exp v,
$$

and hence $f_{*}(v)+s(v)=v$, or $v=\left(1-f_{*}\right)^{-1}(s(v))$.

By the choice of $\lambda_{0},\left(1-f_{*}\right)^{-1} \circ s$ is a lipschitz map of $\left(X_{b}(M),\|\cdot\|_{b}\right)_{2 \lambda_{0}}$ into itself with the lipschitz constant $L\left(\left(1-f_{*}\right)^{-1} \circ s\right) \leqq 1 / 2$. Hence by the contraction principle $v$ must be 0 i.e. $u$ must be the identity map of $M$. Now, take any $x, y \in M$ with $x \neq y$. Put $\operatorname{Per}(f)=\{x \in M \mid x$ is a periodic point of $f\}$.

Case 1: the case of $x \notin \operatorname{Per}(f)$ or $y \notin \operatorname{Per}(f)$. Suppose $x \notin \operatorname{Per}(f)$.

We can define a map $u: M \rightarrow M$ as following:

For any $z \in M$

$$
u(z)= \begin{cases}f^{n}(y) & \text { if }{ }^{\exists} n \text { with } z=f^{n}(x) \\ z & \text { otherwise. }\end{cases}
$$

Then it is clear that $f \circ u=u \circ f$ and that $u \neq 1_{M}$. By (c) we have $d_{0}\left(u, 1_{M}\right)>2 \cdot \lambda_{0}$. Hence there exists an integer $n$ with $d\left(f^{n}(x), f^{n}(y)\right)>\lambda_{0}$. The case of $y \notin \operatorname{Per}(f)$ is similar.

Case II: the case of $x \in \operatorname{Per}(f)$ and $y \in \operatorname{Per}(f)$. Let $r$ and $s$ be the smallest periods of $x$ and $y$ respectively. Suppose $r=s$. We can define a map $u: M \rightarrow M$ as following:

For any $z \in M$

$$
u(z)= \begin{cases}f^{n}(y) & \text { if }{ }^{\mathrm{a}} n \text { with } z=f^{n}(x) \\ z & \text { otherwise. }\end{cases}
$$

It is clear that $f \circ u=u \circ f$ and $u \neq 1_{M}$. By (c) we have $d_{0}\left(u, 1_{M}\right)>2 \lambda_{0}$. By the definition of $u$ we conclude that there exists an integer $n$ with $d\left(f^{n}(x), f^{n}(y)\right)=d_{0}\left(u, 1_{M}\right)>2 \cdot \lambda_{0}>\lambda_{0}$. Suppose $r>s$. We can define a map $u: M \rightarrow M$ as follows:

For any $z \in M$

$$
u(z)= \begin{cases}f^{s+n}(x) & \text { if }{ }^{a} n \text { with } z=f^{n}(x) \\ z & \text { otherwise. }\end{cases}
$$

It is clear that $f \circ u=u \circ f$. Since $x \neq f^{s}(x), u \neq 1_{M}$. Hence we have $d_{0}\left(u, 1_{M}\right)>2 \cdot \lambda_{0}$. By the definition of $u$ there exists an integer $n$ with 
$d\left(f^{n}(x), f^{s+n}(x)\right)>2 \cdot \lambda_{0}$. As $f^{n}(y)=f^{s+n}(y)$ we have

$$
d\left(f^{n}(x), f^{n}(y)\right)+d\left(f^{s+n}(y), f^{s+n}(x)\right) \geqq d\left(f^{n}(x), f^{s+n}(x)\right)>2 \cdot \lambda_{0} .
$$

Hence $d\left(f^{n}(x), f^{n}(y)\right)>\lambda_{0}$ or $d\left(f^{n+s}(x), f^{n+s}(y)\right)>\lambda_{0}$.

The case of $r<s$ is similar.

q.e.d.

For each $g \in L(M)$ with $d_{0}\left(g \circ f^{-1}, 1_{M}\right)<\lambda_{1}$ we define $d_{\ell}(g, f)$ by $d_{\ell}(g, f)=d_{\ell}\left(g \circ f^{-1}, 1_{M}\right)$. (Note that $C^{1}$-diffeomorphism on $M$ is a lipeomorphism on $M$.)

THEOREM. Assume that $f$ is an Anosov diffeomorphism. Then there exists a positive number $\varepsilon_{0}$ satisfying the followihg condition. For any $\varepsilon: 0<\varepsilon<\varepsilon_{0}$ there exists a positive number $\delta=\delta(\varepsilon)$ with the property that for each $g \in L(M)$ with $d_{\ell}(g, f)<\delta$ there exists a unique homeomorphism $u: M \rightarrow M$ such that $g \circ u=u \circ f$ and $d_{0}\left(u, 1_{M}\right)<\varepsilon$.

Proof. Put $K=\left|f^{*}\right|+\operatorname{Sup}_{0<<_{<} \varepsilon_{5}} L_{2}(\varepsilon) . \quad K$ is finite by Lemma 3-3. For each $v \in\left(X^{0}(M),|\cdot|\right)_{\varepsilon_{5}}^{\circ}$ we have

$$
\left|f_{*}(v)+s(v)\right| \leqq\left|f_{*}\right||v|+L_{2}(|v|)|v| \leqq K|v| \text {. }
$$

Choose a positive number $\varepsilon_{6}$ with $\varepsilon_{6} \leqq \operatorname{Min}\left\{\varepsilon_{5}, \varepsilon_{4} / K\right\}$. From Lemma 3-2 and 3-3 we have

$$
\exp w \circ f \circ \exp v \circ f^{-1}=\exp \left\{w+f_{*}(v)+s(v)+r\left(w: f_{*}(v)+s(v)\right)\right\}
$$

for any $w \in\left(X_{\ell}(M),|\cdot|_{\ell}\right)_{\delta_{1}}^{\circ}$ and $v \in\left(X^{0}(M),|\cdot|\right)_{\varepsilon_{8}}^{\circ}$. We may assume that $\left\|w+f_{*}(v)+s(v)+r\left(w: f_{*}(v)+s(v)\right)\right\|<\lambda_{2}$ by making $\delta_{1}$ and $\varepsilon_{6}$ sufficiently small. From the above expression we see that

$$
\exp w \circ f \circ \exp v \circ f^{-1}=\exp v
$$

holds if and only if

$$
w+f_{*}(v)+s(v)+r\left(w: f_{*}(v)+s(v)\right)=v .
$$

As $f$ is Anosov, $1-f_{*}$ is a linear automorphism. Hence the above equality is equivalent to

$$
\left(1-f_{*}\right)^{-1}\left(w+s(v)+r\left(w: f_{*}(v)+s(v)\right)\right)=v .
$$

Put $F(v)=f_{*}(v)+s(v)$ and $G_{w}(v)=\left(1-f_{*}\right)^{-1}\left(w+s(v)+r\left(w: f_{*}(v)+s(v)\right)\right)$. By (ii) in Lemma 3-2 and by (ii) in Lemma 3-3 we have

$$
|r(w: F(v))| \leqq L_{1}\left(|w|_{\ell}, K|v|\right) K|v|
$$


and $|s(v)| \leqq L_{2}(|v|)|v|$. Hence by (iii) in Lemma 3-2 and by (iii) in Lemma 3-3 we can choose positive numbers $\delta_{2}: 0<\delta_{2} \leqq \delta_{1}$ and $\varepsilon_{7}: 0<\varepsilon_{7} \leqq \varepsilon_{6}$ with the property that for each $w \in\left(X_{\ell}(M),|\cdot|_{\ell}\right)_{\delta_{2}}^{\circ}$ and $v \in\left(X^{0}(M),|\cdot|_{\ell 7}\right.$ we have

$$
\left|\left(1-f_{*}\right)^{-1}(r(w: F(v)))\right| \leqq 1 / 3|v|
$$

and

$$
\left|\left(1-f_{*}\right)^{-1}(s(v))\right| \leqq 1 / 3|v|
$$

On the other hand for each $w \in\left(X_{\ell}(M),|\cdot|_{\ell}\right)_{\delta_{1}}^{\circ}$ and $v, v^{\prime} \in\left(X_{0}(M),|\cdot|\right)_{\ell_{6}}^{\circ}$, putting $\delta=|w|_{\ell}$ and $\varepsilon=\operatorname{Max}\left\{|v|,\left|v^{\prime}\right|\right\}$, we have

$$
\begin{aligned}
\left|G_{w}(v)-G_{w}\left(v^{\prime}\right)\right| \leqq & \left|\left(1-f_{*}\right)^{-1}\right|\left\{\left|s(v)-s\left(v^{\prime}\right)\right|+\mid r(w: F(v))\right. \\
& \left.-r\left(w: F\left(v^{\prime}\right)\right) \mid\right\} \\
\leqq & \left|\left(1-f_{*}\right)^{-1}\right|\left\{L_{2}(\varepsilon)\left|v-v^{\prime}\right|+L_{1}(\delta, K \varepsilon)\left(\left|f_{*}\right| \cdot\left|v-v^{\prime}\right|\right.\right. \\
& \left.\left.+L_{2}(\varepsilon)\left|v-v^{\prime}\right|\right)\right\} \\
\leqq & \left|\left(1-f_{*}\right)^{-1}\right|\left\{L_{2}(\varepsilon)+K L_{1}(\delta, K \varepsilon)\right\}\left|v-v^{\prime}\right| .
\end{aligned}
$$

Hence by (ii) in Lemma 3-2 and by (iii) in Lemma 3-3 we can choose positive numbers $\delta_{3}: 0<\delta_{3} \leqq \delta_{1}$ and $\varepsilon_{8}: 0<\varepsilon_{8} \leqq \varepsilon_{6}$ such that for each $w \in\left(X_{\ell}(M),|\cdot|_{\ell}\right)_{\delta_{3}}^{\circ}$ and $v, v^{\prime} \in\left(X^{0}(M),|\cdot|\right)_{\varepsilon_{8}}^{\circ}$ we have

$$
\left|G_{w}(v)-G_{w}\left(v^{\prime}\right)\right| \leqq 1 / 2\left|v-v^{\prime}\right|
$$

For the convenience we may assume that $\delta_{3} \leqq \delta_{2}$ and $\varepsilon_{8} \leqq \varepsilon_{7}$. Now, take any positive number $\varepsilon$ with $0<\varepsilon<\varepsilon_{8}$. For this $\varepsilon$ we can choose a positive number $\delta^{\prime}$ such that for each $w \in\left(X_{\ell}(M),|\cdot|_{\ell}\right)_{\delta^{\prime}}^{\circ}$ we have

$$
\left|\left(1-f_{*}\right)^{-1}(w)\right|<1 / 3 \varepsilon .
$$

Hence, putting $\delta=\operatorname{Min}\left\{\delta^{\prime}, \delta_{3}\right\}$, we have the following

(i) $\left|G_{w}(v)\right|<\varepsilon$ for any $w \in\left(X_{\ell}(M),|\cdot|_{\ell}\right)_{\delta}^{\circ}$ and $v \in\left(X^{0}(M),|\cdot|\right)_{\varepsilon}$

(ii) $\left|G_{w}(v)-G_{w}\left(v^{\prime}\right)\right| \leqq 1 / 2\left|v-v^{\prime}\right|$

for any $w \in\left(X_{\ell}(M),|\cdot|_{\ell}\right)_{\delta}^{\circ}$ and $v, v^{\prime} \in\left(X^{0}(M),|\cdot|\right)_{\varepsilon}$

And so by the contraction principle

(iii) for any $w \in\left(X_{\ell}(M),|\cdot|_{\ell}\right)_{\delta}$ there exists a unique $v \in X^{0}(M)$ such that $|v|<\varepsilon$ and $G_{w}(v)=v$ i.e.

$$
\exp w \circ f \circ \exp v \circ f^{-1}=\exp v \text {. }
$$

Note that $\exp v$ is onto since $\exp v$ is homotopic to the identity. Hence 
the proof of theorem is complete except for proving the injectivity of $u=\exp v$, remarking several facts that for any $g \in L(M)$ and $u \in C^{0}(M)$ $g \circ u=u \circ f$ if and only if $\left(g \circ f^{-1}\right) \circ f \circ u \circ f^{-1}=u$, that if $d_{\ell}(g, f)$ is sufficiently small there exists a unique $w \in X_{\ell}(M)$ with $|w|_{\ell}$ sufficiently small such that $g \circ f^{-1}=\exp w$ (see Prop. 2-2), that if $d_{0}\left(u, 1_{M}\right)$ is sufficiently small there exists a unique $v \in X^{0}(M)$ with $|v|$ sufficiently small such that $u=\exp v$ and that $|\cdot|$ and $\|\cdot\|$ are equivalent. To prove the injectivity let $g$ be a lipeomorphism of $M$ and $u$ be in $C^{0}(M)$ with $d_{0}\left(u, 1_{M}\right)<\lambda_{0} / 2$ and assume $g \circ u=u \circ f$. Choose $x, y \in M$ with $u(x)=$ $u(y)$. If $x \neq y$ there exists an integer $n_{0}$ such that $d\left(f^{n_{0}}(x), f^{n_{0}}(y)\right) \geqq \lambda_{0}$ by Lemma 3-4. As $g^{n_{0}} \circ u=u \circ f^{n_{0}}$ we have $u \circ f^{n_{0}}(x)=g^{n_{0}} \circ u(x)=g^{n_{0}} \circ u(y)$ $=u \circ f^{n_{0}}(y)$. On the other hand as $d_{0}\left(u, 1_{M}\right)<\lambda_{0} / 2$ and $d\left(f^{n_{0}}(x), f^{n_{0}}(y)\right)$ $\geqq \lambda_{0}$ we have $u \circ f^{n_{0}}(x) \neq u \circ f^{n_{0}}(y)$. This is a contradiction. Hence $x=y$.

q.e.d.

\section{REFERENCES}

[1] Anosov, Geodesic flow on a Riemannian manifold with negative curvature, Trudy Math. Just. Stekholv, Moscow, 1967.

[2] Dieudonné, Foundations of modern analysis, Academic Press, New York, 1960.

[3] Hirsch and Pugh, Stable manifolds and hyperbolic sets, Proc. of Symposia in Pure Math. (Global Analysis) XIX, AMS (1970), 133-163.

[4] Moser, On a theorem of Anosov, J. of differential equations 5 (1969), 411-440.

[ 5 ] Nitecki, Differentiable dynamics, Cambridge, M.I.T. Press, 1971.

Department of Mathematics

Nagoya University 